\title{
Energy Aware Performance Comparison of Routing Protocols for Mobile Ad Hoc Networks
}

\author{
Humaira Nishat \\ CVR College of Engineering, Department of ECE, Ibrahimpatan, R.R.District, A.P., India \\ Email:huma_nisha@yahoo.com
}

\begin{abstract}
Mobile nodes in an ad hoc wireless network are battery operated. Thus, it is essential to find energy conserving mechanisms and protocols that optimize the use of battery power in order to increase the lifetime of the network. When finding routes, energy aware routing increases the network lifetime. In this paper, I have considered three routing protocols DSDV, DSR \& AODV for mobile ad hoc networks and evaluated the energy performance metrics in all the four modes- transmitting, receiving, idle and sleep along with other metrics such as residual energy, throughput, packet delivery fraction and end-to-end delay. I have observed that idle energy consumption is responsible for a large portion of the overal energy consumption in the wireless interfaces of the mobile nodes. Finally, by the observation I have concluded that DSR offers the best combination of energy consumption, residual energy of nodes and delay while AODV gives better packet delivery fraction and DSDV gives more throughput.
\end{abstract}

Index Terms- AODV, DSDV, DSR, delay, energy consumption, packet delivery fraction, throughput

\section{INTRODUCTION}

Mobile ad hoc networks [MANETs][1] are wireless networks in which mobile nodes communicate with each other using multi-hop wireless links without any infrastructure support. Nodes are free to move, therefore the network experiences rapid and unpredictable topology changes [2]. Developing routing protocols for MANETs has been an extensive research area in recent years, and many proactive and reactive protocols have been proposed from a variety of perspectives [3], [4], [5]. These protocols try to satisfy various properties, like distributed implementation, efficient energy utilization, throughput optimization, fast route convergence and freedom from loops. Since the nodes are powered by battery, development of energy efficient protocols is needed. When a node exhausts its available energy, it ceases to function and the lack of mobile hosts results in breakdown of network, thereby affecting the overall communication performance.

All the layers of communication are coupled in power consumption and solutions addressing the power saving issue include transmission power control aware routing and low power modes at the physical layer, MAC layer and at the network layer. Routing protocols may balance power consumption at nodes according to their routing decisions. In the present work, we measure and compare the energy consumption behavior of three routing protocols- DSDV, AODV and DSR along with other performance metrics.
The rest of the paper is organized as follows:

Section 2 presents the related work focusing the various energy efficient routing protocols. Section 3 deals with the three routing protocols DSDV, DSR \& AODV. Section 4 gives the simulation setup. In section 5 we present our simulation results and finally section 6 concludes the paper.

\section{RELATED WORK}

A large number of recent studies focus on mobile ad hoc networks [MANETs] [6]. Several studies have dealt with measuring energy consumption in the wireless interfaces of mobile nodes [7], [8] to determine the exact sources of energy consumption in the wireless interfaces. In order to address the energy efficiency issues within ad hoc networks, it is essential to understand the energy model which represents the power consumption behavior in the ad hoc network node wireless interfaces [9]. In AFECA [Adaptive Fidelity Energy Conservation Algorithm], nodes sleep based on the size of their neighborhoods [10]. When number of neighbors is more, then node enters into sleep state without disconnecting the network. [10] provides different sleep patterns for mobile nodes based on their residual energy and quality of service. Techniques to combine power management and power control for wireless cards is presented in [11]. In power conserving algorithm [12], a node enters into the doze state if it overhears RTS/CTS for data transmission. In power saving mode of 802.11 [13] when a node transmits or receives an ad hoc traffic indication (ATIM) frame during an ATIM window, it must be in active mode during the entire beacon interval that results in a much higher energy consumption. [14], [15] compare energy consumption of various reactive and proactive routing protocols under similar traffic. This paper presents a comparison analysis of three routing protocols considering energy consumption as the basic metric and tells which protocol is best suited for increasing the lifetime of the network.

\section{DESCRIPTION OF ROUTING PROTOCOLS FOR MOBILE AD HOC NETWORKS}

\section{A. Destination Sequenced Distance Vector (DSDV)}

Destination Sequenced Distance Vector Routing (DSDV)[19] is a table-driven routing scheme for ad hoc mobile networks based on the Bellman-Ford algorithm. It was developed by C. Perkins and P.Bhagwat in 1994. The main contribution of the algorithm was to solve the 
routing loop problem. The stations periodically transmit their routing tables to their immediate neighbors. The routing table updates are sent either in "full dump" or in "incremental update". A full dump sends the full routing table to the neighbors and could span many packets. When the network is relatively stable, incremental updates are sent to avoid extra traffic. DSDV is quite suitable for creating ad hoc networks with small number of nodes. As it requires a regular update of its routing tables, it uses more battery power and a small amount of bandwidth even when the network is idle.

\section{B. Ad Hoc On-Demand Distance Vector Routing (AODV)}

AODV[20] is an on demand algorithm, meaning that it builds routes between nodes only as desired by source nodes. It uses traditional routing tables, one entry per destination. AODV builds routes using a route request / route reply query cycle. When a source node desires a route to a destination for which it does not already have a route, it broadcasts a route request (RREQ) packet across the network. Nodes receiving this packet update their information for the source node and set up backwards pointers to the source node in the route tables. In addition to the source node's IP address, current sequence number, and broadcast ID, the RREQ also contains the most recent sequence number for the destination of which the source node is aware. A node receiving the RREQ may send a route reply (RREP) if it is either the destination or if it has a route to the destination with corresponding sequence number greater than or equal to that contained in the RREQ. If this is the case, it unicasts a RREP back to the source. Otherwise, it rebroadcasts the RREQ. Nodes keep track of the RREQ's source IP address and broadcast ID. If they receive a RREQ which they have already processed, they discard the RREQ and do not forward it. As the RREP propagates back to the source, nodes set up forward pointers to the destination. Once the source node receives the RREP, it may begin to forward data packets to the destination.

A route is considered active as long as there are data packets periodically traveling from the source to the destination along that path. Once the source stops sending data packets, the links will time out and eventually be deleted from the intermediate node routing tables. If a link break occurs while the route is active, the node upstream of the break propagates a route error (RERR) message to the source node to inform it of the now unreachable destination(s). After receiving the RERR, if the source node still desires the route, it can reinitiate route discovery.

\section{Dynamic Source Routing (DSR)}

The key feature of DSR [18] is the use of source routing. That is, the sender knows the complete hop-by hop route to the destination. These routes are stored in a route cache. The data packets carry the source route in the packet header. When a node in the ad hoc network attempts to send a data packet to a destination for which it does not already know the route, it uses a route discovery process to dynamically determine such a route. Route discovery works by flooding the network with route request (RREQ) packets. Each node receiving a RREQ rebroadcasts it, unless it is the destination or it has a route to the destination in its route cache. Such a node replies to the RREQ with a route reply (RREP) packet that is routed back to the original source. RREQ and RREP packets are also source routed. The RREQ builds up the path traversed so far. The RREP routes itself back to the source by traversing this path backwards. The route carried back by the RREP packet is cached at the source for future use. If any link on a source route is broken, the source node is notified using a route error (RERR) packet. The source removes any route using this link from its cache. A new route discovery process must be initiated by the source, if this route is still needed. DSR makes very aggressive use of source routing and route caching.

\section{IV.SIMULATION SETUP}

I have used network simulator (NS-2.34) for the work. NS2 is a discrete event driven simulator [16],[17] developed at the University of Berkeley and the Virtual Inter Network Testbed (VINT) project 1997. I have used Ubuntu 9.04 linux environment. NS2 is suitable for designing new protocols, comparing different protocols and traffic evaluations. It is an object oriented simulation written in $\mathrm{C}++$, with OTCL interpreter as a frontend.

My simulation setup is a network with randomly placed nodes within an area of $1000 \mathrm{~m} * 1000 \mathrm{~m}$ for a simulation time of 100 second. The parameters used for carrying out simulation are summarized in table1.

\begin{tabular}{|c|c|}
\hline \multicolumn{2}{|c|}{ TABLE.I } \\
\hline Parameter & Value \\
\hline Routing Protocols & DSDV,AODV\& DSR \\
\hline MAC Layer & 802.11 \\
\hline Terrain Size & $1000 \mathrm{~m} * 1000 \mathrm{~m}$ \\
\hline No. of Nodes & $10,20,30,40,50$ \\
\hline Packet Size & $512 \mathrm{~B}$ \\
\hline Initial Energy & 1000Joules \\
\hline Idle Power Consumption & $1.0 \mathrm{~W}$ \\
\hline Rx Power Consumption & $1.0 \mathrm{~W}$ \\
\hline Tx Power Consumption & $1.0 \mathrm{~W}$ \\
\hline Transition Power Consumption & $0.2 \mathrm{~W}$ \\
\hline Simulation Time & $100 \mathrm{~s}$ \\
\hline Traffic Source & TCP \\
\hline
\end{tabular}


The goal of this simulation is to evaluate the performance differences of the three routing protocols in terms of energy consumption, throughput, delay and packet delivery fraction using TCP as traffic source.

\section{A. Performance Metrics}

Average Energy Consumption: It is the average energy consumed by all nodes in transmitting, receiving, idle and sleep mode.

Average Residual Energy: It is the average remaining energy of each node in a network by the end of simulation.

Packet Delivery Fraction: It is the ratio of the number of packets received by the destination to the number of data packets generated by the source.

Average End-to-End Delay: It is the average time taken by the data packets to propagate from source to destination across a MANET. It includes all possible delays caused by buffering during routing discovery latency, queuing at the interface queue and retransmission delays at the MAC propagation and transfer times.

Throughput: It is the ratio of successfully transmitted data packets per second in the network during the simulation.

\section{SIMULATION RESULTS}

Here I present a comparative analysis of the performance metrics of the three routing protocols DSDV, AODV and DSR using TCP traffic source for varying number of nodes $10,20,30,40 \& 50$.

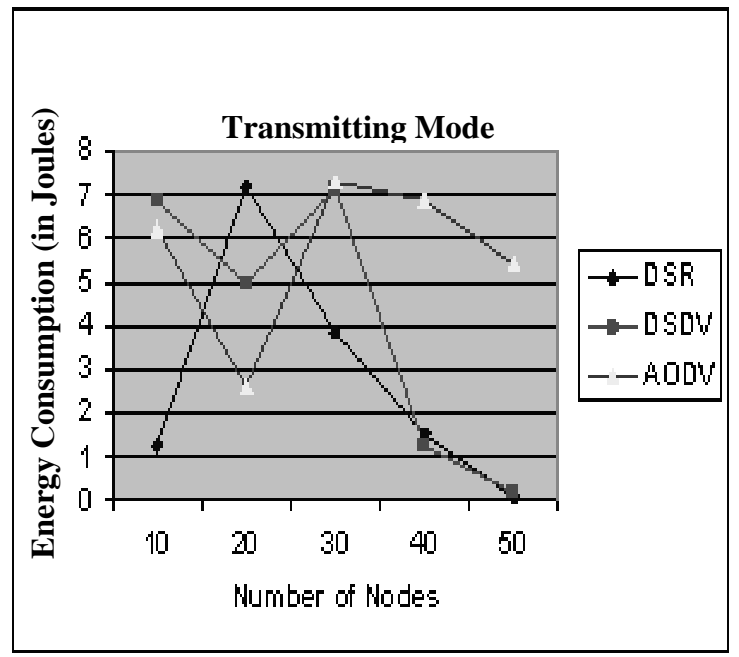

Figure 1.Energy consumption (in Joules) in transmitting mode

Energy consumption in transmitting mode in case of DSR for a network size of 10 is very less compared to that of AODV \& DSDV, but as the size grows to 20, energy consumption increases. At this stage AODV gives the least energy consumption. But as the size grows further to $30,40 \& 50$, DSR performs better than the other two.

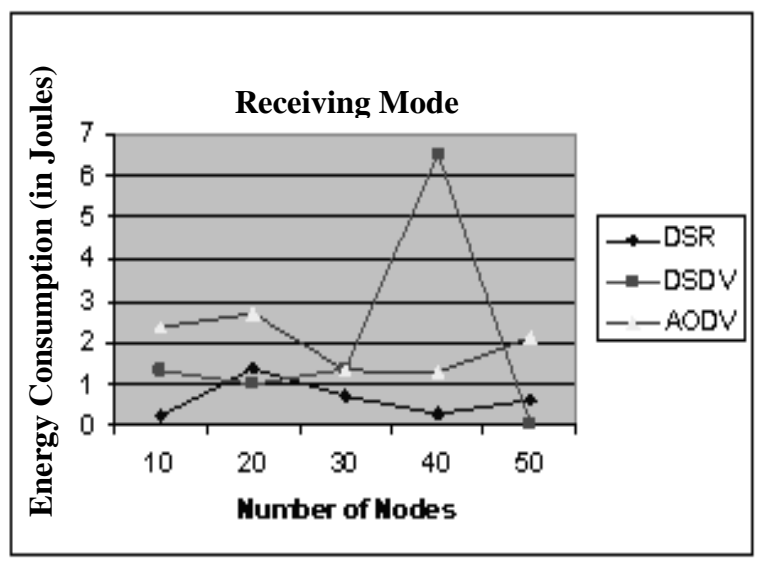

Figure 2. Energy consumption in receiving mode

The amount of energy consumed in receiving mode is almost same as the energy consumed in transmitting mode. From figure 2, it is clear that the energy consumed by DSR is less and is almost constant for a variable network size.

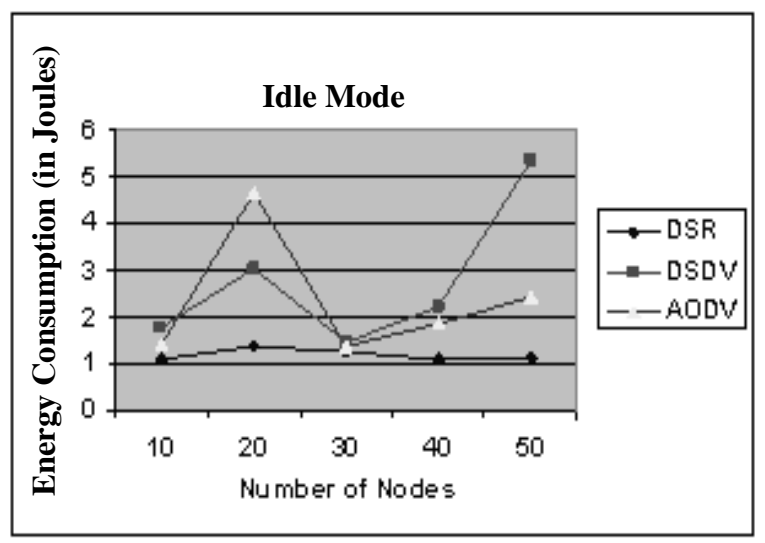

Figure 3. Energy consumption (in Joules) in idle mode

In the idle mode the nodes does not actually communicate but consumes a considerable amount of energy. The amount of energy consumed in idle mode approaches the amount that is consumed in the receiving mode by the destination node. From figure 3, it is clear that energy consumed by nodes in the idle mode is more for AODV \& DSDV than DSR and constitutes almost one-third of the energy consumption.

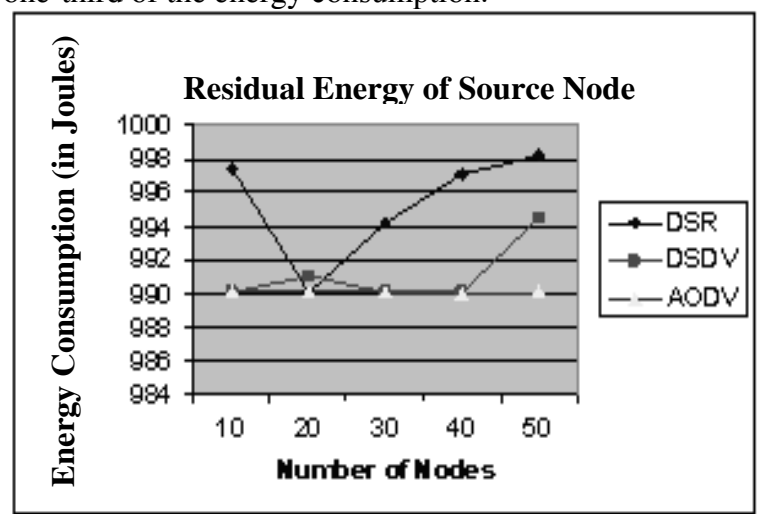

Figure 4. Residual energy (in Joules) of source node 
From figure 4, it is clear that the residual energy of source node is far more for DSR than AODV or DSDV. Also as the size of network increases, residual energy of source node increases for DSR. AODV gives the least amount of remaining energy. Hence, DSR outperforms both DSDV and AODV.

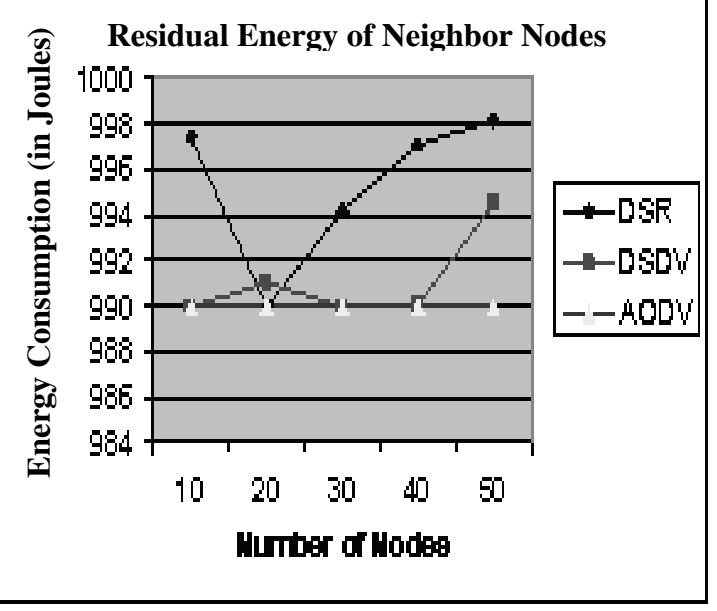

Figure 5. Residual energy (in Joules) of neighbor nodes

Figure 5, gives the amount of energy remaining with the neighbor nodes and destination node at the end of simulation. For the same network setup with three protocols, it is clear from figure 5 that DSR gives the maximum amount of residual energy and AODV the least. Hence, DSR outperforms DSDV and AODV.

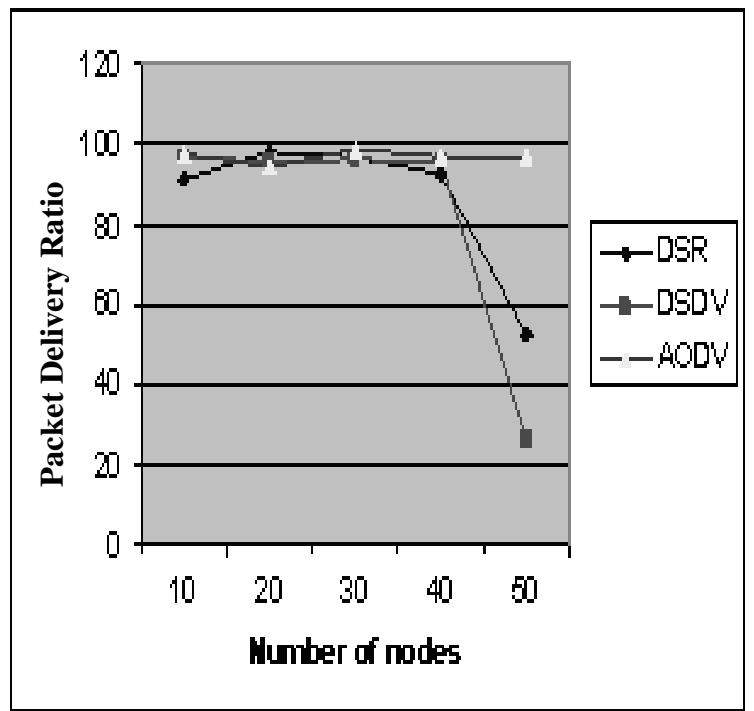

Figure 6. Packet delivery ratio vs number of nodes

Packet delivery fraction is same for all the three routing protocols for a network size of $10,20,30 \& 40$ nodes. But as the network size increases to 50, AODV outperforms both DSR and DSDV. Among DSR \& DSDV, DSR gives better PDF.

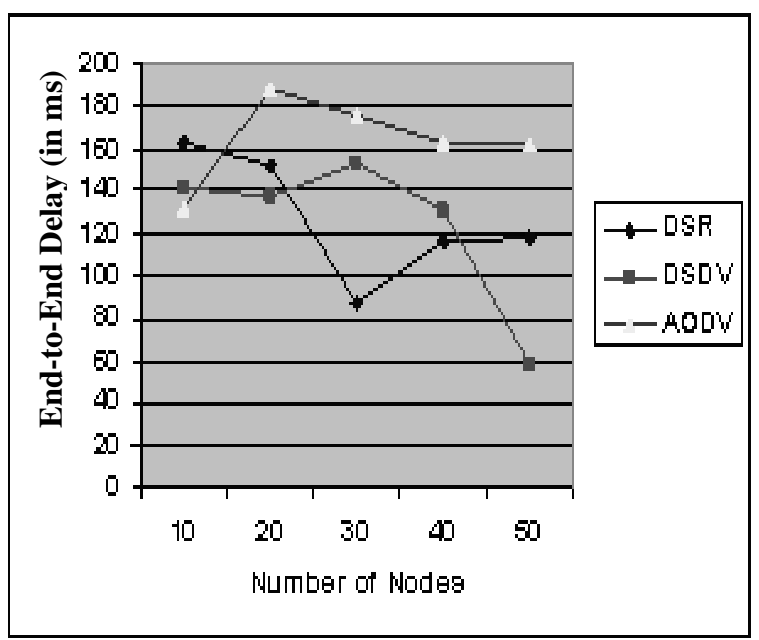

Figure 7. End-to-end delay (in milliseconds) vs number of nodes

End-to-End delay is the average time a packet takes to traverse the network. Initially, the end-to-end delay of AODV is less compared to DSR \& DSDV with 10 nodes but with the increased network size the end-to-end delay decreases for DSR thereby outperforming both AODV and DSDV.

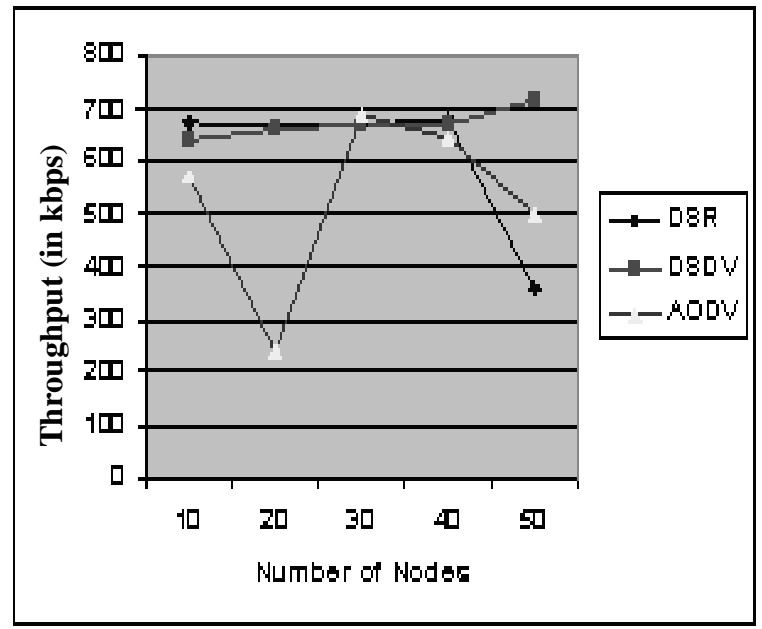

Figure 8. Throughput (in kbps) vs number of nodes Based on the simulation results, it is clear that the throughput value of DSR \& DSDV is almost constant and more compared to AODV. For a network size of 50, DSDV gives better throughput performance than DSR and AODV.

\section{CONCLUSIONS}

In this work I have analyzed the energy consumption behavior of both proactive and reactive routing protocolsDSDV, DSR \& AODV for ad hoc networks using network simulator. I have evaluated and showed how the different modes (transmitting, receiving, idle and sleep) of energy consumption affect the energy usage of mobile devices. I found that the amount of energy consumed in idle mode i.e., due to overhearing by neighboring nodes 
is equal to the amount of energy consumed by a destination node in receiving mode. Also, due to the caching strategy used by reactive routing protocols such as DSR, DSR is more likely to find a route in the cache and hence resorts to route discovery less frequently than AODV and DSDV resulting in more residual energy. There is no energy consumption by nodes in the sleep mode. Overall, the energy consumed by nodes in all modes is very less for DSR than AODV. Also the residual energy is more for DSR than the other two routing protocols.

This paper also compares the performance of the three protocols in terms of PDR, throughput \& delay. From the simulation results I conclude that DSR gives better combination of energy consumption, residual energy and delay among the three protocols. AODV gives more packet delivery fraction and DSDV gives better throughput. Future work will focus on new algorithms and approaches to extend the lifetime of nodes in a network.

\section{REFERENCES}

[1] C.K.Toh "Ad Hoc Mobile Wireless Networks Protocols and Systems", First Edition, Prentice Hall Inc, USA 2002.

[2] N.H.Vaidya, "Mobile Ad Hoc Networks Routing MAC and Transport Issues", Proceedings of the IEEE International Conference on Computer Communication INFOCOM, 2004.

[3] E.Royer \& C.K.Toh, " A Review of Current Routing Protocols for Ad hoc Mobile Wireless Networks", IEEE Personal Communications Magazine, Vol.6, No.2, April 1999.

[4] C.K. Toh, "Associativity Based Routing For Ad Hoc Mobile Networks", Wireless Personal Communication Journal, Special Issue on Mobile Networking and Computing Systems, Vol.4, No.2, March 1997.

[5] V.D. Park, M.S.Carson, "A Highly Adaptive Distributed Routing Algorithm For Mobile Wireless Networks", IEEE IIFOCOM, Kobe, Japan, 1997.

[6] Internet Engineering Task Force, "MANET Working Group Charter", http:// www.ictf.org/html.charters/manetcharter.html.

[7] L.M.Freeney, "An Energy Consumption Model for Performance Analysis of Routing Protocols for Mobile Ad Hoc Networks", Mobile Networks and Applications, Volume 6, Issue 3, June 2001, Pages 239-249.

[8] L.M Freeney and M.Nilson, "Investigating the Energy Consumption of a Wireless Network Interface in an Ad Hoc Networking Environment", Proceedings of IEEE INFOCOM 2001, Volume 3, April 2001, Pages 1548-1557.

[9] R.Kravets and P. Krishnan, "Power Management Techniques for Mobile Communications", Proceedings of the ACM Mobile Computing and Networking Conference, Dallas Texas, October 1998, Pages 157-168.

[10] Y.Xu.J.Heidemann and D. Estrin, "Adaptive Energy Conserving Routing For Multihop Ad Hoc Networks", Technical Report 527, USC/Information Services Institute, 2000.

[11] T.Simunic, H.Vikalo, P.Glynn, G.D.Micheli, "Energy Efficient Design of Portable Wireless Systems", Proceedings of the International Symposium an Low Power Electronics and Design, 2000, Pages 49-54.

[12] Juan Carlos Cano and Pietro Manzoni, "Evaluating the Energy Consumption Reduction in a MANET by
Dynamically Switching-off Network Interfaces" Proc. of the $6^{\text {th }}$ IEEE Symposium on Computers and Communications, July 2001.

[13] Eun-Sun Jung and Nitin H.Vaidya, "An Energy Efficient MAC Protocol for Wireless LANs", IEEE INFOCOM 2002, New York, USA, June 2002.

[14] Humaira Ehsan and Zartash Afzal Uzmi, "Performance Comparison of Ad Hoc Wireless Network Routing Protocols", Proceedings of INMIC 2004, $8^{\text {th }}$ International Multitopic Conference, pp 457-465, IEEE December 2004.

[15] Charles E.Perkins and Pravin Bhagwat, "Highly Dynamic Destination Sequenced Distance Vector Routing (DSDV) for Mobile Computers", Proceedings of ACM SIGCOMM, oct 1994.

[16] UCB/LBNL/VINT Network Simulator http://www.mash.cs.berkeley.edu/ns/referred on March 2010.

[17] "The Network Simulator -ns-2", available at http://www.isi.edu/nsnam/ns/referred on March 2010.

[18] Josh Broch, David Johnson, and David Maltz. "The Dynamic Source Routing Protocol For Mobile Ad Hoc Networks", http://www.ietf.org/internet-drafts/ drafts-ietfmanet-dsr-01.txt, Dec 1998. IETF Internet Draft(work in progress)

[19] Charle E. Perkins and Pravin Bhagwat. "Highly Dynamic Destination Sequenced Distance Vector Routing (DSDV) for Mobile Computers" 1994.

[20] Charles Perkins and Elizabeth Royer "Ad Hoc On-demand Distance Vector Routing", In Proceedings of the $2^{\text {nd }}$ IEEE Workshop on Mobile Computing Systems and Applications, Pages 90-100, Feb 1999. 